\title{
Prolonged Mechanical Ventilation: Are You a Lumper or a Splitter?
}

In this issue of RespiRATORy CARE, Kojicic and colleagues describe long-term survival in patients who underwent tracheostomy for anticipated or prolonged mechanical ventilation (PMV) in Olmsted County, Minnesota. ${ }^{1}$ The study confirmed that approximately $10 \%$ of patients with invasive mechanical ventilation undergo tracheotomy and that overall 1-year mortality in that population is still substantial. Older age and the presence of COPD negatively impacted survival, whereas survival was better among neuromuscular patients who continued to receive mechanical ventilation even after discharge. Importantly, Kojicic et al note that chronic conditions that relate to the underlying cause of mechanical ventilation should be taken into account when considering PMV outcomes.

\section{See the Original Study on Page 1765}

Early on in medical training, one becomes familiar with the concept of "lumpers and splitters" when classifying and treating medical diseases. A lumper takes on an expansive view of the problem in order to gain generality. Lumping patients into broad categories facilitates large, multicenter trials; however, important differences between subgroups of the population may be lost. In contrast, a splitter focuses on details to gain precision about a problem. In research, splitting may result in a limited number of patients in a category, thus making it more difficult to study them. When discussing PMV, one could take the approach of a lumper or a splitter. There are advantages and disadvantages to both tactics.

The study of pulmonary disease processes has benefited from analyzing a population as whole and then by indentifying differences among subgroups of the population. For example, the Acute Respiratory Distress Syndrome Network trial was a large, multicenter trial that found a significant reduction in mortality and ventilator days in the group treated with lower tidal volumes. ${ }^{2}$ That study had an important impact on how patients with ARDS are ventilated in the intensive care unit (ICU). However, subsequent studies identified important heterogeneity in the ARDS patient population. Certain subgroups, such as those with trauma-related ARDS, have better outcomes, whereas those with ARDS from sepsis fare worse. ${ }^{3,4}$ While larger studies are necessary to provide statistical power when answering a specific question, they may not fully reflect individual patient complexity.

The lack of a uniformly accepted definition of PMV has impacted how these patients are studied. The definitions of PMV have depended on whether the population is identified for clinical, research, or payment purposes. Several definitions are used to describe PMV, including the duration of need for mechanical ventilation, and simply the presence of a tracheostomy, which implies the need for PMV. ${ }^{5-8}$ The earlier timing of tracheostomy placement may also impact the definition of the PMV population and study outcomes. ${ }^{8-10}$ Should we combine patients who require mechanical ventilation continuously for 24 hours per day with those who only require nocturnal mechanical ventilation when studying PMV outcomes? Important findings relevant to subpopulations of PMV patients may not be apparent if the PMV population is studied as a whole (ie, lumping). Thus, it is important to recognize how PMV patients are identified when drawing inferences from studies.

PMV patients are cared for in a variety of settings. Due to financial pressures on ICU resource utilization, stable patients who require PMV are often transferred to alternative care settings, including step-down respiratory care units and long-term acute-care hospitals that specialize in a multidisciplinary approach to weaning and rehabilitation. ${ }^{11,12}$ Increasing numbers of PMV patients and their families are electing to continue care at home, which requires substantial coordination of care and adjustment. ${ }^{13-15}$ Studies performed in the ICU tend to influence how patients undergoing PMV are cared for in the post-acute-care setting. For example, a target hemoglobin concentration of $\geq 7 \mathrm{~g} / \mathrm{dL}$ is recommended for mechanically ventilated patients in the ICU, to minimize transfusion-associated complications. ${ }^{16}$ As a result, a restrictive transfusion strategy has been applied to PMV patients outside of the ICU. However, the optimal transfusion strategy for patients weaning from PMV is unknown. ${ }^{17,18}$

Weaning is another example of how ICU-developed protocols influence long-term acute-care hospital practice. The use of a standardized weaning protocol in the ICU has helped streamline the weaning process, reduced the number of days to extubation, decreased the need for tracheostomy, and lowered ICU costs. ${ }^{19}$ Weaning protocols have also reduced weaning times in PMV patients at long-term acute-care hospitals. ${ }^{20}$ Nonetheless, not all patients are suitable for a weaning protocol, and an individualized approach is necessary in patients with higher complexity. ${ }^{21}$ Thus, more work is needed to guide the care of PMV patients in the long-term acute-care hospital setting.

Patients require PMV for a variety of reasons, including obstructive lung disease, infectious or non-infectious lung injury, neuromuscular disease (including critical illness neuromyopathy), and postoperative respiratory failure. One-year survival of PMV patients ranges from $23 \%$ to $76 \%$ and underscores the problems that clinicians face when talking to 


\section{Prolonged Mechanical Ventilation: Are You a Lumper or a Splitter?}

patients and their surrogates about long-term prognosis. ${ }^{5}$ Many PMV patients have underlying comorbidities, and successful weaning from PMV does not guarantee long-term survival. ${ }^{22}$ A recent prospective study found that PMV patients had multiple transitions within post-discharge care facilities, resulting in profound disability and high financial costs. ${ }^{23}$ The lack of a uniform database to track PMV patients throughout the healthcare continuum contributes to the difficulties in studying this patient population. The functional recovery and ultimate survival of patients undergoing PMV tend to be overestimated by both physicians and patient surrogates. Furthermore, surrogate decision makers for PMV patients reported that few physicians discussed what to expect about the patient's future survival, general health, and care-giving needs. ${ }^{24}$ Involvement of palliative care should be incorporated into the comprehensive care of PMV patients, in an effort to align expectations and improve communication between physicians and patients and their surrogates. ${ }^{5,22}$ Additional longitudinal studies will provide physicians with muchneeded data to help guide the care of PMV patients throughout the healthcare continuum.

Future studies are needed to better understand how the definitions of PMV impact study outcomes and patient care. Outcome studies on subgroups of PMV patients will help physicians personalize care for these complex patients and may better align expectations between caregivers and patients and their surrogates. Comparative effectiveness research will also help better define the role of the post-acute-care setting in the care of these patients. In summary, whether you are a lumper or a splitter, more work is needed to optimize the care of ICU survivors who require PMV.

Heidi H O'Connor MD

Pulmonary and Sleep Medicine

New England Sinai Hospital Stoughton, Massachusetts

\section{REFERENCES}

1. Kojicic M, Li G, Ahmed, A, Thakur L, Trillo-Alvarez C, CartinCeba $\mathrm{R}$, et al. Long-term survival in patients with tracheostomy and prolonged mechanical ventilation in Olmsted County, Minnesota. Respir Care 2011;56(11):1765-1770.

2. The Acute Respiratory Distress Syndrome Network. Ventilation with lower tidal volumes as compared with traditional tidal volumes for acute lung injury and the acute respiratory distress syndrome. N Engl J Med 2000;342(18):1301-1308.

3. Calfee CS, Eisner MD, Ware LB, Thompson BT, Parsons PE, Wheeler AP, et al. Trauma-associated lung injury differs clinically and biologically from acute lung injury due to other clinical disorders. Crit Care Med 2007;35(10):2243-2250.

The author has disclosed no conflicts of interest.

Correspondence: Heidi H O'Connor MD, Pulmonary and Sleep Medicine, New England Sinai Hospital, 150 York Street Stoughton MA 02072. E-mail: hoconnor@nesinai.org.

DOI: $10.4187 /$ respcare. 01600
4. Sheu CC, Gong MN, Zhai R, Chen F, Bajwa EK, Clardy PF, et al. Clinical characteristics and outcomes of sepsis-related vs non-sepsisrelated ARDS. Chest 2010;138(3):559-567.

5. MacIntyre NR, Epstein SK, Carson S, Scheinhorn D, Christopher K, Muldoon S; National Association for Medical Direction of Respiratory Care. Management of patients requiring prolonged mechanical ventilation: report of a NAMDRC consensus conference. Chest 2005; 128(6):3937-3954.

6. Gillespie DJ, Marsh HM, Divertie MB, Meadows JA. Clinical outcome of respiratory failure in patients requiring prolonged (greater than 24 hours) mechanical ventilation. Chest 1986;90(3):364-369.

7. Gracey DR, Viggiano RW, Naessens JM, Hubmayr RD, Silverstein MD, Koenig GE. Outcomes of patients admitted to a chronic ventilator-dependent unit in an acute-care hospital. Mayo Clin Proc 1992; 67(2):131-136.

8. Cox CE, Carson SS, Holmes GM, Howard A, Carey TS. Increase in tracheostomy for prolonged mechanical ventilation in North Carolina, 1993-2002. Crit Care Med 2004;32(11):2219-2226.

9. Quintel M, Roth H. Tracheostomy in the critically ill: clinical impact of new procedures. Intensive Care Med 1999;25(3):326-328.

10. Scheinhorn DJ, Chao DC, Stearn-Hassenpflug M, LaBree LD, Heltsley DJ. Post-ICU mechanical ventilation: treatment of 1,123 patients at a regional weaning center. Chest 1997;111(6):1654-1659.

11. Scheinhorn DJ, Hassenpflug MS, Votto JJ, Chao DC, Epstein SK, Doig GS, et al. Post-ICU mechanical ventilation at 23 long-term care hospitals: a multicenter outcomes study. Chest 2007;131(1):85-93.

12. Scheinhorn DJ, Hassenpflug MS, Votto JJ, Chao DC, Epstein SK, Doig GS, et al. Ventilator-dependent survivors of catastrophic illness transferred to 23 long-term care hospitals for weaning from prolonged mechanical ventilation. Chest 2007;131(1):76-84.

13. Goldstein RS, Psek JA, Gort EH. Home mechanical ventilation. Demographics and user perspectives. Chest 1995;108(6):1581-1586.

14. Wright SE, VanDahm K. Long-term care of the tracheostomy patient. Clin Chest Med 2003;24(3):473-487.

15. Divo MJ, Murray S, Cortopassi F, Celli BR. Prolonged mechanical ventilation in Massachusetts: the 2006 prevalence survey. Respir Care 2010;55(12):1693-1698.

16. Hebert PC, Wells G, Blajchman MA, Marshall J, Martin C, Pagliarello $\mathrm{G}$, et al. A multicenter, randomized, controlled clinical trial of transfusion requirements in critical care. Transfusion Requirements in Critical Care Investigators, Canadian Critical Care Trials Group. N Engl J Med 1999;340(6):409-417.

17. Hebert PC, Blajchman MA, Cook DJ, Yetisir E, Wells G, Marshall J, Schweitzer I. Do blood transfusions improve outcomes related to mechanical ventilation? Chest 2001;119(6):1850-1857.

18. Silver MR. Anemia in the long-term ventilator-dependent patient with respiratory failure. Chest 2005;128(5 Suppl 2):568S-575S.

19. Boles JM, Bion J, Connors A, Herridge M, Marsh B, Melot C, et al. Weaning from mechanical ventilation. Eur Respir J 2007;29(5):10331056.

20. Scheinhorn DJ, Chao DC, Stearn-Hassenpflug M, Wallace WA. Outcomes in post-ICU mechanical ventilation: a therapist-implemented weaning protocol. Chest 2001;119(1):236-242.

21. O'Connor HH, White AC. Tracheostomy decannulation. Respir Care 2010;55(8):1076-1081.

22. Nelson JE, Cox CE, Hope AA, Carson SS. Chronic critical illness. Am J Respir Crit Care Med 2010;182(4):446-454.

23. Unroe M, Kahn JM, Carson SS, Govert JA, Martinu T, Sathy SJ. One-year trajectories of care and resource utilization for recipients of prolonged mechanical ventilation: a cohort study. Ann Intern Med 2010;153(3):167-175.

24. Cox CE, Martinu T, Sathy SJ, Clay AS, Chia J, Gray AL. Expectations and outcomes of prolonged mechanical ventilation. Crit Care Med 2009;37(11):2888-2894. 